\title{
What is the shape of an air bubble on a liquid surface?
}

Article

Accepted Version

Teixeira, M. A. C., Arscott, S., Cox, S. J. and Teixeira, P. I. C. (2015) What is the shape of an air bubble on a liquid surface? Langmuir, 31 (51). pp. 13708-13717. ISSN 0743-7463 doi: https://doi.org/10.1021/acs.langmuir.5b03970 Available at https://centaur.reading.ac.uk/48224/

It is advisable to refer to the publisher's version if you intend to cite from the work. See Guidance on citing.

Published version at: http://dx.doi.org/10.1021/acs.langmuir.5b03970

To link to this article DOI: http://dx.doi.org/10.1021/acs.langmuir.5b03970

Publisher: American Chemical Society

All outputs in CentAUR are protected by Intellectual Property Rights law, including copyright law. Copyright and IPR is retained by the creators or other copyright holders. Terms and conditions for use of this material are defined in the End User Agreement.

\section{www.reading.ac.uk/centaur}

\section{CentAUR}

Central Archive at the University of Reading

Reading's research outputs online 


\title{
What is the shape of an air bubble on a liquid surface?
}

\author{
Miguel A. C. Teixeira* \\ Department of Meteorology, University of Reading \\ Earley Gate, PO Box 243, Reading RG6 6BB, United Kingdom* \\ Steve $\operatorname{Arscott}^{\dagger}$ \\ Institut d'Electronique, de Microélectronique et de Nanotechnologie (IEMN) \\ CNRS UMR8520, The University of Lille \\ Cité Scientifique, Avenue Poincaré, 59652 Villeneuve d'Ascq, France ${ }^{\dagger}$ \\ Simon J. Cox \\ Department of Mathematics, Aberystwyth University \\ Aberystwyth, Ceredigion, SY23 3BZ, United Kingdom ${ }^{\ddagger}$ \\ Paulo I. C. Teixeira ${ }^{\S}$ \\ ISEL - Instituto Superior de Engenharia de Lisboa, Instituto Politécnico de Lisboa \\ Rua Conselheiro Emídio Navarro 1, 1959-007 Lisbon, Portugal and \\ Centro de Física Teórica e Computacional, \\ Faculdade de Ciências da Universidade de Lisboa \\ Campo Grande, Edifício C8, 1749-016 Lisbon, Portugals
}

(Dated: 24 November 2015) 


\begin{abstract}
We have calculated the equilibrium shape of the axially symmetric meniscus along which a spherical bubble contacts a flat liquid surface, by analytically integrating the Young-Laplace equation in the presence of gravity, in the limit of large Bond numbers. This method has the advantage that it provides semi-analytical expressions for key geometrical properties of the bubble in terms of the Bond number. Results are in good overall agreement with experimental data and are consistent with fully numerical (Surface Evolver) calculations. In particular, we are able to describe how the bubble shape changes from hemispherical, with a shallow flat bottom, to lenticular, with a deeper, curved bottom, as the Bond number is decreased.

PACS numbers: 83.80.Iz, 82.70.Rr
\end{abstract}

*Electronic address: M.A.Teixeira@reading.ac.uk

${ }^{\dagger}$ Electronic address: steve.arscott@iemn.univ-lille1.fr

${ }^{\ddagger}$ Electronic address: sxc@aber.ac.uk

$\S$ Electronic address: piteixeira@fc.ul.pt 


\section{INTRODUCTION}

A fundamental understanding of the behaviour of bubbles and liquid films is very important for several fields of industry such as mining, manufacturing, materials, security, and food production $[1,2]$. In addition to these better-known sectors, the potential use of bubbles and films has been recently demonstrated in emerging technological domains such as micro [3-7] and nanotechnologies [8-10]. Soap bubbles and films have been studied for many years $[11,12]$, with more recent studies concerning their organisation $[13,14]$, electrification [5, 15], magnetization [16], wetting [18-20], stability [21-23], aerodynamics [24] and their mechanical [25] and optical properties [26].

In this paper, we study the wetting of a sessile soap bubble on a liquid surface of the same soap solution. This was first addressed by Nicolson [27], who derived an analytical approximation for the shape of the meniscus surrounding a single, axisymmetric air bubble on a liquid surface, by integrating the Young-Laplace equation. However, in so doing Nicolson linearised the curvature term, which effectively restricts the applicability of his solutions to bubbles that protrude little from the liquid surface, i.e., small bubbles (in the sense of small Bond number as will be defined below). Later, Howell [28] solved the same problem for arbitrarily large curvature in two dimensions (2d), and, in the limit of small bubbles (small Bond number), for axisymmetric bubbles in three dimensions (3d). Here we go beyond these approaches and consider the $3 \mathrm{~d}$ axisymmetric bubble problem for a wide range of Bond numbers (i.e., bubble sizes). In particular we are interested in describing the crossover between large bubbles with nearly hemispherical films and flat bottoms, and small bubbles with lenticular films and curved bottoms. We also wish to assess how important it is to fully take into account the $3 \mathrm{~d}$ nature of a bubble. This extends our earlier work [19] on the contact angle of bubbles on solid surfaces of different wettabilities: an air bubble on a free liquid surface will of course wet the surface completely, but will also be more or less immersed beneath the surface.

This paper is organised as follows: in section II we describe our experimental method for measuring bubble shapes. Then in section III we present our model, the Young-Laplace equation for the meniscus around a single spherical bubble on a liquid surface, and obtain closed-form expressions for the inner and outer meniscus surfaces, as well as a relationship between the film inclination at the top of the meniscus and the meniscus height. An alter- 
native method to find bubble shapes from numerical energy minimisation, using the Surface Evolver software, is described in section IV. Then in section V we compare our experimental results with the predictions of our semi-analytical solution of the Young-Laplace equation, as well as with the fully numerical Surface Evolver solution. Finally, we conclude in section VI.

\section{EXPERIMENTAL}

The bubble wetting experiments were performed in a class ISO $5 / 7$ cleanroom which ensures that the temperature $(T)$ and relative humidity $(R H)$ remain within the following ranges: $T=20 \pm 0.5^{\circ} \mathrm{C}$ and $R H=45 \pm 2 \%$. The photographic data were gathered using a contact angle meter (GBX Scientific Instruments, France). A commercially available soap solution (Pustefix, Germany) was used to generate bubbles for the experiments. This solution is a mixture of pure water, a second liquid (glycerol) to increase the viscosity of the solution and reduce drainage, and a surfactant (an organosulphate). The surface tension of the commercial solution was measured to be $28.2 \pm 0.3 \mathrm{~mJ} \mathrm{~m}^{-2}$ using the pendant drop method [29] and applying a correction factor [30]. This measured value is comparable with other experiments concerning soap bubbles and soap films [17, 18].

The experimental set-up, shown in figure 1a, consists of a $10 \mathrm{~mm}$ deep transparent Perspex container containing the commercial soap solution. The Perspex container was filled to the brim using the soap solution in order to form a convex meniscus at the surface. This enabled the meniscus around the bubble to be clearly visible to the side-mounted cameras. Air-filled sessile soap bubbles having base radii in the range $0.5-40 \mathrm{~mm}$ were generated for the experiments using a pipette (Bio-Rad, France) having a tip diameter of $0.5 \mathrm{~mm}$. Pendant bubbles were first generated using the pipette - these bubbles were then brought into contact with the soap solution surface where they wet the surface and become sessile bubbles once the pipette is retracted. The sessile bubbles attain their stable sessile shape rapidly (in less than 1 second) and are stable on the liquid surface for 10-30 seconds - enough time for photographs to be taken. Following the collapse of the bubble, the liquid surface of the bath was carefully skimmed in order to remove any daughter bubbles formed. The bath was then refilled to the brim in order to recreate the convex meniscus.

As the bubbles are macroscopic - millimetres to centimetres in size - the determination 
of the bubble dimensions (base radius $r$, height $H$, meniscus height $h$ ) is accurate using imaging. The experimental setup also allows the bottom of the bubble to be visible to the side-mounted camera, so the bubble depth $d$ can be measured as well. Finally, the apparent bubble contact angle $\theta_{b}$ - the inclination at the top of the meniscus - is given by $\theta_{b}=2 \tan ^{-1}(H / r)$, where all quantities are defined in figure $1 \mathrm{~b}$.

The experiments involve many bubbles (around 80 in total). The bubble base diameters (as defined in figure 1b) vary from $1 \mathrm{~mm}$ to $8 \mathrm{~cm}$. However, the experimental technique (i.e., pipetting the bubbles manually) results in obtaining several bubbles having virtually the same size. These raw data can be plotted (e.g., figure 7a), which involves a certain scatter. In order to obtain the error bars in figure $7 \mathrm{~b}$, the raw data can be sorted (in terms of increasing contact angle). The $h / R$ data can then be "binned" for contact angles which fall within $20^{\circ}-24^{\circ}, 25^{\circ}-29^{\circ}, 30^{\circ}-34^{\circ}$, etc. This enables error bars for $\theta_{b}$ and $h / R$ to be obtained.

\section{THEORETICAL MODEL}

The starting point for our model is the Young-Laplace equation for the shape of the meniscus around an axisymmetric bubble, which may be written [12]:

$$
\left[1+\left(\frac{d x}{d z}\right)^{2}\right]^{-3 / 2}\left[-\frac{d^{2} x}{d z^{2}}+\frac{1+\left(\frac{d x}{d z}\right)^{2}}{x}\right]=\frac{\Delta p}{\gamma},
$$

where $z$ is the height and $x$ is the distance to the film surface from its axis of symmetry (here located at the $z$ axis, see figure 2). $\Delta p$ is the pressure difference across the surface (inner minus outer) and $\gamma$ is the surface tension of the liquid. The left-hand side of equation (1) is just the curvature of the liquid-air surface inside or outside the bubble. The bubble film is a partial spherical shell. which we assume to be of constant, infinitesimal thickness. The meniscus, on the other hand, comprises an outer and an inner surface; equation (1) must be solved separately for each of these.

This equation cannot be solved analytically except in a very few special cases. A numerical solution is complicated by the fact that one of the boundary conditions is defined at infinity: $x(z=0)=+\infty$ for the outer meniscus surface or, which is more physically transparent, $z(x=+\infty)=0$ - the fluid surface is flat infinitely far from the bubble. The equivalent boundary condition for the inner meniscus surface $d z / d x(x=0)=0$ - the liquid surface 
is horizontal at the bottom of the bubble. One more boundary condition is needed at the point where the inner and outer meniscus surfaces meet the bubble film: the inner and outer meniscus surfaces must be tangent. The angle that they make with the horizontal is unknown a priori and finding it is one of the main objects of the present calculations.

In order to circumvent these difficulties, we adopt a parametric representation of equation (1) in terms of the film inclination. We first obtain the shape of the inner and outer meniscus surfaces, and then proceed to derive a relation between the angle at the top of the meniscus (the bubble contact angle) and either the Bond number, or the ratio of meniscus height to bubble film curvature radius (henceforth referred to as "bubble radius"). These are two possible measures of bubble size.

Let $\theta$ be the angle, measured on a vertical plane containing the $z$ axis, between the tangent to the film and the horizontal axis (see figure 2): clearly $\cot \theta=-d x / d z$. Furthermore, the outer surface of the meniscus meets the free surface of the liquid outside the bubble at an angle $\theta=0$, and the inner surface of the meniscus meets the liquid surface at the centre of the bubble at an angle $\theta=\pi$. Finally, the apparent contact angle of the bubble, defined as the film inclination at the top of the meniscus, where the inner and outer surfaces are tangent, is defined as $\theta_{b}=\theta(z=h$ ) (see figure 2). Replacing the dependent variable $x$ by $\theta$ in equation (1), this takes the form

$$
\left(-\frac{d \theta}{d z}+\frac{1}{R \sin \theta_{b}+\int_{z}^{h} \cot \theta d z}\right) \sin \theta=\frac{\Delta p}{\gamma},
$$

where $R$ is the bubble radius. Applying equation (2) at the inner and outer surfaces of the meniscus yields

$$
\begin{aligned}
\left(\frac{d \theta}{d z}-\frac{1}{R \sin \theta_{b}+\int_{z}^{h} \cot \theta d z}\right) \sin \theta=\frac{p_{o}-p_{b}}{\gamma} \quad \text { (outer) } \\
\left(-\frac{d \theta}{d z}+\frac{1}{R \sin \theta_{b}+\int_{z}^{h} \cot \theta d z}\right) \sin \theta=\frac{p_{i}-p_{b}}{\gamma} \quad \text { (inner), }
\end{aligned}
$$

where $p_{i}, p_{o}$ and $p_{b}$ are the pressures inside the bubble, outside the bubble, and inside the meniscus, respectively. The pressure inside the meniscus is assumed to be in hydrostatic equilibrium, such that

$$
p_{b}=p_{b 0}-\rho g z
$$

where $p_{b 0}$ is the pressure at the liquid free surface outside the bubble, $g$ is the acceleration due to gravity and $\rho$ is the density of the liquid. Moreover, the pressure difference between 
the inside and the outside of the bubble is given by

$$
p_{i}-p_{o}=4 \frac{\gamma}{R}
$$

In their present form, equations (3) and (4) still cannot be solved analytically. However, they can be made more tractable by rewriting them in such a way that $z$ becomes the dependent variable and $\theta$ the independent variable. Changing the integration variable in the integral from $z$ to $\theta$,

$$
\int_{z}^{h} \cot \theta d z=\int_{\theta}^{\theta_{b}} \cot \theta^{\prime} \frac{d z}{d \theta^{\prime}} d \theta^{\prime}
$$

where $\theta^{\prime}$ is a dummy integration variable and we have used the fact that, on the surfaces bounding the meniscus, $\theta$ is a monotonic function of $z$. Further, using equation (5), equations (3) and (4) take the form

$$
\begin{aligned}
& \left(\frac{p_{o}-p_{b 0}}{\gamma}+\frac{\rho g z}{\gamma}+\frac{\sin \theta}{R \sin \theta_{b}+\int_{\theta}^{\theta_{b}} \cot \theta^{\prime} \frac{d z}{d \theta^{\prime}} d \theta^{\prime}}\right) \frac{d z}{d \theta}=\sin \theta \quad \text { (outer), } \\
& \left(\frac{p_{i}-p_{b 0}}{\gamma}+\frac{\rho g z}{\gamma}-\frac{\sin \theta}{R \sin \theta_{b}+\int_{\theta}^{\theta_{b}} \cot \theta^{\prime} \frac{d z}{d \theta^{\prime}} d \theta^{\prime}}\right) \frac{d z}{d \theta}=-\sin \theta \quad \text { (inner). }
\end{aligned}
$$

Note that, in the first of these equations, the first term in the brackets vanishes, as $p_{o}=p_{b 0}$ at a planar liquid surface.

At this point, it is useful to non-dimensionalise the equations, so that the orders of magnitude of the various terms become clearer. $\theta$ is dimensionless by nature, and takes values of $\mathcal{O}(1)$. Now bubble shape is determined by the balance of gravity and surface tension, so a key dimensionless quantity is the Bond number, defined as

$$
\mathrm{Bo}=\frac{\rho g R^{2}}{\gamma}
$$

The relevant lengthscale is the capillary length $\lambda_{c}=(\gamma / \rho g)^{1 / 2}$; for the soap solution used $\left(\gamma=28.2 \mathrm{~mJ} \mathrm{~m}^{-2}, g=9.8 \mathrm{~m} \mathrm{~s}^{-2}, \rho=1010 \mathrm{~kg} \mathrm{~m}^{-3}\right)$, this is $\lambda_{c} \approx 1.7 \mathrm{~mm}$. The dimensionless vertical coordinate is thus defined as $z^{\prime}=z / \lambda_{c}$. In terms of these new variables, equations (8) and (9) become

$$
\begin{gathered}
\left(z^{\prime}+\mathrm{Bo}^{-1 / 2} \frac{\sin \theta}{\sin \theta_{b}+\mathrm{Bo}^{-1 / 2} \int_{\theta}^{\theta_{b}} \cot \theta^{\prime} \frac{d z^{\prime}}{d \theta^{\prime}} d \theta^{\prime}}\right) \frac{d z^{\prime}}{d \theta}=\sin \theta \quad \text { (outer), } \\
\left(4 \mathrm{Bo}^{-1 / 2}+z^{\prime}-\mathrm{Bo}^{-1 / 2} \frac{d z^{\prime}}{\sin \theta}=-\sin \theta \quad\right. \text { (inner), }
\end{gathered}
$$


where equations (6) and (10) have been used. In the limit of large Bond numbers, Bo $\gg 1$ (gravity-dominated regime), we can expand the fraction containing the integral in equations (11) and (12) to first order in $\mathrm{Bo}^{-1 / 2}$, with the results

$$
\begin{gathered}
{\left[z^{\prime}+\mathrm{Bo}^{-1 / 2} \frac{\sin \theta}{\sin \theta_{b}}\left(1-\mathrm{Bo}^{-1 / 2} \frac{1}{\sin \theta_{b}} \int_{\theta}^{\theta_{b}} \cot \theta^{\prime} \frac{d z^{\prime}}{d \theta^{\prime}} d \theta^{\prime}\right)\right] \frac{d z^{\prime}}{d \theta}=\sin \theta \quad \text { (outer), }} \\
{\left[4 \mathrm{Bo}^{-1 / 2}+z^{\prime}-\mathrm{Bo}^{-1 / 2} \frac{\sin \theta}{\sin \theta_{b}}\left(1-\mathrm{Bo}^{-1 / 2} \frac{1}{\sin \theta_{b}} \int_{\theta}^{\theta_{b}} \cot \theta^{\prime} \frac{d z^{\prime}}{d \theta^{\prime}} d \theta^{\prime}\right)\right] \frac{d z^{\prime}}{d \theta}=-\sin \theta \quad \text { (inner). }}
\end{gathered}
$$

The terms of $\mathcal{O}(1)$ in these equations account for the vertical curvature of the meniscus, the terms of $\mathcal{O}\left(\mathrm{Bo}^{-1 / 2}\right)$ for the horizontal curvature (i.e. the axisymmetry), and the terms of $\mathcal{O}\left(\mathrm{Bo}^{-1}\right)$ for the difference in distances between the inner and outer surfaces of the meniscus to the axis of symmetry of the bubble. Therefore, the terms of $\mathcal{O}\left(\mathrm{Bo}^{-1 / 2}\right)$ and $\mathcal{O}\left(\mathrm{Bo}^{-1}\right)$ (which are small if Bo $\gg 1$ ) account for $3 \mathrm{~d}$ geometrical effects.

As we shall see below, these equations still provide a good approximation even for fairly small Bond numbers. The advantage of taking the large-Bond-number limit is that it allows the solutions to be written as power series in $\mathrm{Bo}^{-1 / 2}$ :

$$
z^{\prime}=z_{0}^{\prime}+z_{1}^{\prime} \mathrm{Bo}^{-1 / 2}+z_{2}^{\prime} \mathrm{Bo}^{-1}+\ldots
$$

and we can then systematically solve for $z_{i}^{\prime}$ up to the desired order of approximation.

We have thus obtained the height $z$ of the meniscus surface as a function of its inclination $\theta$. In order to completely specify the meniscus surface, we need to find $x$, the distance of a point on the surface to the symmetry axis, also as a function of $\theta$. To this we now turn.

Let $\Delta x=x\left(\theta_{b}\right)-x(\theta)$ be the distance, measured horizontally, from a point on the surface to the top of the meniscus $-\Delta x$ is negative outside the bubble, as $x$ is a radial coordinate and therefore always positive. It follows from the definition of $\theta$ that

$$
\Delta x^{\prime}=\int_{\theta_{b}}^{\theta} \cot \theta^{\prime} \frac{d z^{\prime}}{d \theta^{\prime}} d \theta^{\prime}
$$

where we have non-dimensionalised $x$ as we did $z$ before, i.e., $\Delta x^{\prime}=\Delta x / \lambda_{c} . \Delta x^{\prime}$ can likewise be expanded in a power series of $\mathrm{Bo}^{-1 / 2}$ :

$$
\Delta x^{\prime}=\Delta x_{0}^{\prime}+\Delta x_{1}^{\prime} \mathrm{Bo}^{-1 / 2}+\Delta x_{2}^{\prime} \mathrm{Bo}^{-1}+\ldots
$$


where

$$
\Delta x_{i}^{\prime}=\int_{\theta_{b}}^{\theta} \cot \theta^{\prime} \frac{d z_{i}^{\prime}}{d \theta^{\prime}} d \theta^{\prime} \quad(i=0,1,2, \ldots) .
$$

The solution procedure is now clear: we start by solving equation (13) (equation (14)) together with equation (15) for the outer (inner) meniscus surface. Results for $z_{0}^{\prime}, z_{1}^{\prime}, z_{2}^{\prime}, \ldots$ are then substituted into equations (18) to find $\Delta x_{0}^{\prime}, \Delta x_{1}^{\prime}, \Delta x_{2}^{\prime}, \ldots$, thereby completely specifying the meniscus shape from equation (17).

We shall now treat the outer and inner surfaces of the meniscus separately. In all cases we shall stop at the second order of approximation (i.e., at $\mathcal{O}\left(\mathrm{Bo}^{-1}\right)$ ), which, as will be seen, provides satisfactory results. Higher-order approximations are feasible but quickly become cumbersome, as they contain increasing numbers of integrals that need to be evaluated numerically. Additionally, Bo must then be obtained as the solution of an algebraic equation of order higher than two, which has a much more complicated form. This is a necessary intermediate step when developing the theory, so that eventually we will be able to evaluate quantities as functions of Bo, which is what is relevant experimentally.

\section{A. Outer surface}

Inserting equation (15) into equation (13) and collecting powers of Bo we obtain the following three equations, valid at zeroth, first- and second-order in $\mathrm{Bo}^{-1 / 2}$ :

$$
\begin{aligned}
& z_{0}^{\prime} \frac{d z_{0}^{\prime}}{d \theta}=\sin \theta \\
& z_{0}^{\prime} \frac{d z_{1}^{\prime}}{d \theta}+\left(z_{1}^{\prime}+\frac{\sin \theta}{\sin \theta_{b}}\right) \frac{d z_{0}^{\prime}}{d \theta}=0 \\
& z_{0}^{\prime} \frac{d z_{2}^{\prime}}{d \theta}+\left(z_{1}^{\prime}+\frac{\sin \theta}{\sin \theta_{b}}\right) \frac{d z_{1}^{\prime}}{d \theta}+\left(z_{2}^{\prime}-\frac{\sin \theta}{\sin ^{2} \theta_{b}} \int_{\theta}^{\theta_{b}} \cot \theta^{\prime} \frac{d z_{0}^{\prime}}{d \theta^{\prime}} d \theta^{\prime}\right) \frac{d z_{0}^{\prime}}{d \theta}=0 .
\end{aligned}
$$

These equations must be solved subject to the boundary conditions $z_{0}^{\prime}(\theta=0)=z_{1}^{\prime}(\theta=0)=$ $z_{2}^{\prime}(\theta=0)=0$, The results are:

$$
\begin{aligned}
& z_{0}^{\prime}=\sqrt{2}(1-\cos \theta)^{1 / 2} \\
& z_{1}^{\prime}=-\frac{4\left(1-\cos ^{3} \frac{\theta}{2}\right)}{3 \sqrt{2} \sin \theta_{b}(1-\cos \theta)^{1 / 2}} \\
& z_{2}^{\prime}=\frac{1}{z_{0}^{\prime}} \int_{0}^{\theta} \frac{\sin \theta^{\prime}}{z_{0}^{\prime}}\left[\frac{\sin \theta^{\prime}}{\sin ^{2} \theta_{b}}\left(\int_{\theta^{\prime}}^{\theta_{b}} \frac{\cos \theta^{\prime \prime}}{z_{0}^{\prime}} d \theta^{\prime \prime}\right)+\frac{1}{z_{0}^{\prime}}\left(z_{1}^{\prime}+\frac{\sin \theta^{\prime}}{\sin \theta_{b}}\right)^{2}\right] d \theta^{\prime}
\end{aligned}
$$


which yield, from equation (18),

$$
\begin{aligned}
\Delta x_{0}^{\prime} & =\int_{\theta_{b}}^{\theta} \frac{\cos \theta^{\prime}}{z_{0}^{\prime}} d \theta^{\prime}=\log \frac{1+\cos \frac{\theta_{b}}{2}}{\sin \frac{\theta_{b}}{2}}-\log \frac{1+\cos \frac{\theta}{2}}{\sin \frac{\theta}{2}}-2 \sin \frac{\theta_{b}}{2}+2 \sin \frac{\theta}{2}, \\
\Delta x_{1}^{\prime} & =-\int_{\theta_{b}}^{\theta} \frac{\cos \theta^{\prime}}{z_{0}^{\prime 2}}\left(z_{1}^{\prime}+\frac{\sin \theta^{\prime}}{\sin \theta_{b}}\right) d \theta^{\prime} \\
\Delta x_{2}^{\prime} & =\int_{\theta_{b}}^{\theta} \frac{\cos \theta^{\prime}}{z_{0}^{\prime 2}}\left[\frac{\sin \theta^{\prime}}{\sin ^{2} \theta_{b}}\left(\int_{\theta^{\prime}}^{\theta_{b}} \frac{\cos \theta^{\prime \prime}}{z_{0}^{\prime}} d \theta^{\prime \prime}\right)-z_{2}^{\prime}+\frac{1}{z_{0}^{\prime}}\left(z_{1}^{\prime}+\frac{\sin \theta^{\prime}}{\sin \theta_{b}}\right)^{2}\right] d \theta^{\prime} .
\end{aligned}
$$

The above equations completely specify the shape of the outer surface of the meniscus. Note

that, in equations (24), (26) and (27), equations (19) and (20) have been used to eliminate $d z_{0}^{\prime} / d \theta$ and $d z_{1}^{\prime} / d \theta$. Additionally, although the inner integrals in equations (24) and (27) can be calculated analytically (they are given by equation (25)), the outer integrals, as well as the integral in equation (26), must be evaluated numerically.

\section{B. Inner surface}

Now, the same procedure must be followed for the inner surface of the meniscus. We obtain the following three equations, again valid at zeroth, first- and second-order in $\mathrm{Bo}^{-1 / 2}$ :

$$
\begin{aligned}
& z_{0}^{\prime} \frac{d z_{0}^{\prime}}{d \theta}=-\sin \theta \\
& z_{0}^{\prime} \frac{d z_{1}^{\prime}}{d \theta}+\left(4+z_{1}^{\prime}-\frac{\sin \theta}{\sin \theta_{b}}\right) \frac{d z_{0}^{\prime}}{d \theta}=0, \\
& z_{0}^{\prime} \frac{d z_{2}^{\prime}}{d \theta}+\left(4+z_{1}^{\prime}-\frac{\sin \theta}{\sin \theta_{b}}\right) \frac{d z_{1}^{\prime}}{d \theta}+\left(z_{2}^{\prime}+\frac{\sin \theta}{\sin ^{2} \theta_{b}} \int_{\theta}^{\theta_{b}} \cot \theta^{\prime} \frac{d z_{0}^{\prime}}{d \theta^{\prime}} d \theta^{\prime}\right) \frac{d z_{0}^{\prime}}{d \theta}=0 .
\end{aligned}
$$

These equations must be solved subject to the boundary conditions $z_{0}^{\prime}(\theta=\pi)=z_{2}^{\prime}(\theta=\pi)=$ $0, z_{1}^{\prime}(\theta=\pi)=-4$. The last of these conditions accounts for the pressure difference between the inside and the outside of the bubble, and is only strictly valid if the inner menisci on opposite sides of the bubble do not overlap at the bottom of the bubble (this is consistent with a large-Bo approximation). The results are:

$$
\begin{aligned}
& z_{0}^{\prime}=\sqrt{2}(1+\cos \theta)^{1 / 2} \\
& z_{1}^{\prime}=-4+\frac{4\left(1-\sin ^{3} \frac{\theta}{2}\right)}{3 \sqrt{2} \sin \theta_{b}(1+\cos \theta)^{1 / 2}}, \\
& z_{2}^{\prime}=\frac{1}{z_{0}^{\prime}} \int_{\pi}^{\theta} \frac{\sin \theta^{\prime}}{z_{0}^{\prime}}\left[-\frac{\sin \theta^{\prime}}{\sin ^{2} \theta_{b}}\left(\int_{\theta^{\prime}}^{\theta_{b}} \frac{\cos \theta^{\prime \prime}}{z_{0}^{\prime}} d \theta^{\prime \prime}\right)-\frac{1}{z_{0}^{\prime}}\left(\frac{\sin \theta^{\prime}}{\sin \theta_{b}}-4-z_{1}^{\prime}\right)^{2}\right] d \theta^{\prime},
\end{aligned}
$$


which yield, from equation (18),

$$
\begin{aligned}
\Delta x_{0}^{\prime} & =-\int_{\theta_{b}}^{\theta} \frac{\cos \theta^{\prime}}{z_{0}^{\prime}} d \theta^{\prime}=-\log \frac{1+\cos \frac{\theta_{b}}{2}}{\sin \frac{\theta_{b}}{2}}+\log \frac{1+\cos \frac{\theta}{2}}{\sin \frac{\theta}{2}}+2 \sin \frac{\theta_{b}}{2}-2 \sin \frac{\theta}{2}, \\
\Delta x_{1}^{\prime} & =\int_{\theta_{b}}^{\theta} \frac{\cos \theta^{\prime}}{z_{0}^{\prime 2}}\left(z_{1}^{\prime}+4-\frac{\sin \theta^{\prime}}{\sin \theta_{b}}\right) d \theta^{\prime}, \\
\Delta x_{2}^{\prime} & =\int_{\theta_{b}}^{\theta} \frac{\cos \theta^{\prime}}{z_{0}^{\prime 2}}\left[-\frac{\sin \theta^{\prime}}{\sin ^{2} \theta_{b}}\left(\int_{\theta^{\prime}}^{\theta_{b}} \frac{\cos \theta^{\prime \prime}}{z_{0}^{\prime}} d \theta^{\prime \prime}\right)+z_{2}^{\prime}-\frac{1}{z_{0}^{\prime}}\left(\frac{\sin \theta^{\prime}}{\sin \theta_{b}}-4-z_{1}^{\prime}\right)^{2}\right] d \theta^{\prime} .
\end{aligned}
$$

The above equations completely specify the shape of the inner surface of the meniscus. As in equations (24) and (27), the inner integrals in equations (33) and (36) are given analytically by equation (34), but the outer integrals, as well as the integral in equation (35), must be evaluated numerically. Still, this is much less computationally expensive than solving the full equations numerically.

Since the zeroth-order solutions presented in this and the preceding section neglect all $3 \mathrm{~d}$ effects, they would correspond to the exact $2 \mathrm{~d}$ solutions of Howell [28], except that they do not account for the pressure difference between the inside and the outside of the bubble, which in the present approach only enters at first order.

\section{Relation between $\theta_{b}$ and $h / R$}

We shall now derive a relationship between the meniscus height $h$ and the film inclination at the top of the meniscus, $\theta_{b}$. This is perhaps the easiest prediction of our theory to verify experimentally.

By definition, $h=z\left(\theta=\theta_{b}\right)$, hence

$$
h^{\prime}=\frac{h}{\lambda_{c}}=z^{\prime}\left(\theta=\theta_{b}\right) \approx z_{0}^{\prime}\left(\theta=\theta_{b}\right)+z_{1}^{\prime}\left(\theta=\theta_{b}\right) \mathrm{Bo}^{-1 / 2}+z_{2}^{\prime}\left(\theta=\theta_{b}\right) \mathrm{Bo}^{-1},
$$

where we have used the same non-dimensionalisation as before, and truncated the expansion at second order. On the other hand, we also have

$$
\frac{h}{R}=h\left(\frac{\rho g}{\gamma}\right)^{1 / 2}\left(\frac{\gamma}{\rho g R^{2}}\right)^{1 / 2}=h^{\prime} \mathrm{Bo}^{-1 / 2},
$$

whence it follows that

$$
\frac{h}{R} \approx z^{\prime}\left(\theta=\theta_{b}\right) \mathrm{Bo}^{-1 / 2}=z_{0}^{\prime}\left(\theta=\theta_{b}\right) \mathrm{Bo}^{-1 / 2}+z_{1}^{\prime}\left(\theta=\theta_{b}\right) \mathrm{Bo}^{-1}+z_{2}^{\prime}\left(\theta=\theta_{b}\right) \mathrm{Bo}^{-3 / 2}
$$


We now note that at the top of the meniscus the inner and outer solutions coincide: $z_{\text {outer }}^{\prime}(\theta=$ $\left.\theta_{b}\right)=z_{\text {inner }}^{\prime}\left(\theta=\theta_{b}\right)$. Equating the expressions for these quantities as power series in $\mathrm{Bo}^{-1 / 2}$, truncated at second order as in equation (39), we find the following second-degree algebraic equation:

$$
\left(z_{\text {outer }, 0}^{\prime}-z_{\text {inner }, 0}^{\prime}\right) \mathrm{Bo}^{1}+\left(z_{\text {outer }, 1}^{\prime}-z_{\text {inner }, 1}^{\prime}\right) \mathrm{Bo}^{1 / 2}+\left(z_{\text {outer }, 2}^{\prime}-z_{\text {inner }, 2}^{\prime}\right)=0,
$$

(where all terms are evaluated at $\theta=\theta_{b}$ ), which can be solved for $\mathrm{Bo}^{1 / 2}$ as a function of $\theta_{b}$ :

$\mathrm{Bo}^{1 / 2}=\frac{-\left(z_{\text {outer }, 1}^{\prime}-z_{\text {inner }, 1}^{\prime}\right)-\sqrt{\left(z_{\text {outer }, 1}^{\prime}-z_{\text {inner }, 1}^{\prime}\right)^{2}-4\left(z_{\text {outer }, 0}^{\prime}-z_{\text {inner }, 0}^{\prime}\right)\left(z_{\text {outer }, 2}^{\prime}-z_{\text {inner }, 2}^{\prime}\right)}}{2\left(z_{\text {outer }, 0}^{\prime}-z_{\text {inner }, 0}^{\prime}\right)}$.

This is the physically meaningful root, i.e., the one that reduces to the first-order approximation when $z_{\text {outer }, 2}^{\prime} \rightarrow 0$ and $z_{\text {inner,2 }}^{\prime} \rightarrow 0$. Substituting this result in equation (39) then yields the desired relationship between $h / R$ and $\theta_{b}$. The calculation is straightforward but the resulting formula is rather involved, so we do not show it here. To obtain the first-order relation equivalent to equation (41), the corresponding solutions must instead be truncated at first order, which amounts to neglecting the last term within brackets on the left-hand side of equation (40).

\section{NUMERICAL METHOD}

An alternative to solving the Young-Laplace equation for the bubble and meniscus shape is to perform a direct numerical minimisation of the surface energy - or, equivalently, of the surface area, as the surface tension is assumed constant - using the Surface Evolver software [31]. Note that the Young-Laplace equation is actually the Euler-Lagrange equation for the surface energy minimisation problem with the bubble volume constrained to be fixed.

As in the preceding section, we assume the bubble to be axisymmetric about $x=0$ and seek the profiles $z(x)$ for the top and bottom of the bubble and the free surface outside the bubble.

The initial datafile for Surface Evolver consists of just four vertices and three edges. Two of the vertices are constrained to move on $x=0$, representing the top and bottom of the centre of the bubble, one vertex is at the top of the meniscus, and the other is fixed at $x=x_{\max }, z=0$, representing the equilibrium position of the liquid far from the bubble (we 
take $x_{\max }=7.0 \mathrm{~cm}$ ). The edge connecting the top of the bubble to the top of the meniscus has tension $\gamma_{2}$ and contributes to the total energy an amount $\gamma_{2} \int 2 \pi x \mathrm{~d} l$. The inner edge connecting the bottom of the bubble to the top of the meniscus has tension $\gamma_{1}$ and contributes to the total energy an amount $\gamma_{1} \int 2 \pi x \mathrm{~d} l$. The total volume of the bubble is the difference in the volume beneath these two lines, which we constrain to a prescribed value. The third, outer, edge, representing the free surface, connects the top of the meniscus to the point at $x=x_{\max }$, with the same energetic contribution as the edge beneath the bubble. The total volume of liquid, which is constrained to a fixed value, is the volume beneath the latter two edges. We take $\gamma_{1}=28.2 \mathrm{~mJ} / \mathrm{m}^{2}, \gamma_{2}=56.2 \mathrm{~mJ} / \mathrm{m}^{2}$ to avoid numerical problems due to a zero contact angle between the two air-liquid interfaces at the top of the meniscus; instead the contact angle there is $5^{\circ}=\cos ^{-1}\left(\gamma_{2} / 2 \gamma_{1}\right)$ in our calculations. We have checked that doubling the contact angle leads to differences of at most $9 \%$ in all the data. We therefore conclude that the results are not particularly sensitive to the value of the contact angle.

At each step, we minimise the total energy of the system using gradient descent and second-derivative information (Hessian) with error bounds for subdivision of edges between $0.01 \mathrm{~cm}$ and $0.05 \mathrm{~cm}$. We measure the length of each interface, the height of the meniscus, the depth of the centre of the bubble, and the angle $\theta_{b}$ at the top of the meniscus. Then the volume of the bubble (equivalently, the Bond number) is increased and this step repeated.

\section{RESULTS AND DISCUSSION}

Figure 3 shows examples of sessile bubbles on a bath of the same soap solution, for a range of bubble sizes. The Bond number measures the relative strengths of the gravitational potential and surface tension contributions to the total energy of a bubble. Thus, small bubbles correspond to small Bond numbers (surface tension dominates and pulls the bubble into the solution in order to minimise the film surface area, and hence its surface energy), whereas large bubbles correspond to large Bond numbers (gravity dominates and the bubble rises out of the solution with very small meniscus, so as to minimise its gravitational potential energy). From photographs of bubbles such as these the parameters defined in figure 1b were measured, as described in section II. 


\section{A. The meniscus around a bubble}

Figures 4 and 5 show, respectively, the dimensionless meniscus height $h / R$ and the apparent contact angle of the bubble $\theta_{b}$, defined as the film inclination at the top of the meniscus, vs Bond number Bo. We have also included the predictions of Howell's 2d and 3d theories [28]. In Howell's $2 \mathrm{~d}$ theory, we have corrected the definition of Bond Number by multiplying it by 4 . This is to account for the fact that, when there is curvature in two dimensions, the equivalent radius of the bubble is twice that when there is curvature in only one dimension, for the same pressure difference between the inside and the outside of the bubble. Since Howell's $2 \mathrm{~d}$ results (figure 3 in [28]) are only available for (uncorrected) Bo $<6$, we only present them here up to a maximum value of (corrected) Bo $\approx 24$. Note that our second-order $h / R$ and $\theta_{b}$ become multi-valued at $\mathrm{Bo} \approx 3$, where the large-Bo approximation employed to derive them is clearly questionable; only the upper branch of the curves is physically meaningful. The first-order approximation to $\theta_{b}$ is also multi-valued. Howell's $2 \mathrm{~d}$ solutions for $h / R$ and $\theta_{b}$ are clearly in much less good agreement with experiment than either our first-

or second-order approximations (the agreement would be even worse if we had not corrected Howell's 2d solutions as described above). Moreover, Howell's 3d solution for $\theta_{b}$ (though not that for $h / R$ ) lies closer to the experimental data than his $2 \mathrm{~d}$ solution, further highlighting the importance of treating the bubble as a truly 3 d object. For reasons unclear to us, our first-order approximation provides a better description of the Bond number dependence of the apparent contact angle than our second-order approximation.

In figure 6 we combine the data in figures 4 and 5 to plot the apparent contact angle of the bubble vs dimensionless meniscus height $h / R$, a relation which is perhaps the easiest to compare with directly-measured quantities. There is good agreement between experiment and the predictions of our second-order approximation, even for $\theta_{b}$ down to about $45^{\circ}$, corresponding to $\mathrm{Bo} \sim 4$ (see figure 5). As would be expected, the agreement degrades for small values of $\theta_{b}$, more rapidly for the first-order than for the second-order solution. Noteworthy is also the fact that $h / R$ is a non-monotonic function of $\theta_{b}$, with a maximum at $\theta_{b} \sim 50^{\circ}$, which is captured by the theory. Physically, this maximum can be understood as due to the fact that, for relatively small values of $\theta_{b}$ (i.e., small Bo), the meniscus is not vertical but rather strongly tilted, as the bubble is, to a large extent, immersed in the liquid. To join up with the flat surface far away from the bubble, the outer surface of this 
non-vertical meniscus only has to reorient by a relatively small angle, which can be achieved over a relatively small height.

Although our solution method is formally only valid for large Bond numbers, in practice it can be pushed to fairly small (but still larger than 1) Bo without much loss of accuracy. We also show results obtained using the Surface Evolver, which are in even better agreement with experiment. Here the main problem is that, as pointed out in section IV, one also needs to assume a finite angle between the two air-liquid interfaces at the top of the meniscus. This may explain the small discrepancies between these results and the theory at the largest values of $\theta_{b}$, where almost perfect agreement would be expected.

\section{B. The full bubble profile}

To construct the full profile of any bubble we need to represent the inner and outer solutions for the menisci on either side of the bubble, as well as draw the bubble film. The left column of figure 7 shows the first- and second-order inner and outer solutions for each meniscus scaled by the bubble radius $R$, at a horizontal separation consistent with the bubble radius (which is of course one in these units), for a large bubble (large Bo, top row), an intermediate bubble (moderate Bo, middle row) and a small bubble (small Bo, bottom row). When Bo is large, the bubble bottom is flat and the inner solutions of the two menisci blend seamlessly at the centre of the bubble. When Bo is moderate or small, however, the inner solutions intersect at the centre of the bubble at a finite angle, since they have not yet reached their asymptotic limit. This only means that the solutions, as derived here, would not, in principle, be valid for such values of Bo. However, a heuristic way to extend the domain of validity of the theory without reformulating it is simply to add the solutions for the two menisci and subtract the common asymptotic value of the inner solutions. By construction, this allows the film at the bottom of the bubble to have zero slope and be symmetric with respect to the bubble axis, as it should. By following this procedure, the second-order solutions are then added together to produce the full bubble profiles in the right column of figure 7 , which we have overlaid on photographs of actual bubbles with the same $\theta_{b}$. In this comparison, the radii $R$ of the predicted bubbles have been re-scaled to coincide with those of the measured bubbles. Agreement is seen to be very good for large and moderate Bond numbers, but to deteriorate markedly for small Bond numbers. The 
reason for this is twofold. Firstly, the solutions are inherently less accurate for small Bo. Secondly, the construction outlined above is also less accurate for very small bubbles, since the inner solution of any given meniscus is not yet flat when it reaches the meniscus on the opposite side of the bubble. This leads to a noticeable change in $\theta_{b}$ (associated with a discontinuity between the film angle at the top of the menisci and at the bottom of the bubble), to a severe overestimation of the meniscus height, and also to a spurious increase in the height of the whole film, which causes the bubble to portrude excessively from the fluid. This latter feature can be detected more easily if we overlay the meniscus solutions without adding them on the photograph of the small bubble (figure 8): agreement improves considerably, although of course the inner surfaces of the menisci now meet at a finite angle at the centre of the bubble.

\section{Getting to the bottom of the bubble}

It is easily observed that larger bubbles are almost perfect hemispheres sitting atop the liquid surface, whereas smaller bubbles are almost completely immersed, with only a small lenticular film. This is reflected in the shape of the bubble bottom, which is flat in the former case, but almost spherical in the latter. Our theory also allows us to extract the Bond number dependence of the bubble depth, as defined in figures $1 \mathrm{~b}$ and 2 : it equals $|z(x=0)|$, where $z$ is the sum of the inner solutions for the two menisci minus their common asymptotic value. This is plotted in figure 9a as the dotted and solid lines for the first- and second-order solutions, respectively. Both the first- and second-order approximations predict a maximum in $d / R$ vs Bo which is not borne out by experiment. The range of Bo spanned by the experimental data is considerably smaller than in figures 4 and 5, owing to the difficulty in measuring $d$, especially when this quantity is small. We have also calculated $d / R$ assuming that the two menisci do not interfere, i.e., that the inner solution attains its asymptotic depth before reaching the centre of the bubble (which obviously is not realistic for any other than the largest values of Bo). This is given analytically by

$$
\frac{d}{R}=4 \mathrm{Bo}^{-1}
$$

which assumes that the depth to which the bubble penetrates is due only to the pressure difference between the inside and the outside the bubble. This latter approximation clearly 
yields a poorer agreement with experiment.

Although it is not clear whether $d / R$ vs Bond number has a maximum, it is clear that $d$ itself must attain a maximum. Equation (42), valid for large bubbles, may be expressed alternatively as

$$
\frac{d}{\lambda_{c}}=4 \mathrm{Bo}^{-1 / 2}
$$

which predicts that $d / \lambda_{c}$ decreases as Bo increases, and therefore that $d$ also decreases as Bo increases, for fixed values of $g$ and $\gamma$. Since the bubble is always in contact with the surface (owing to buoyancy forces), for small Bo the depth $d$ may never exceed the bubble diameter (which increases as Bo increases). So $d$ - and hence also $d / \lambda_{c}-$ must have a maximum for some intermediate value of Bo (or, equivalently, of the bubble radius $R$, see equation (10)). Figure 9b compares first- and second-order theory, as well as Surface Evolver results, for $d / \lambda_{c}$ vs Bo with experimental data. Although the experimental data do not exhibit a clear maximum for $d / \lambda_{c}$ as there are not enough data points at large Bo, both theory and Surface Evolver predict this maximum very clearly, suggesting that it is $d / \lambda_{c} \approx 1.15$ or $d \approx 0.195 \mathrm{~cm}$ (recall that $\lambda_{c} \approx 0.17 \mathrm{~cm}$ ) for $\mathrm{Bo} \approx 4$. This corresponds to a bubble radius $R \sim 1 \mathrm{~cm}$.

The best agreement with the data is achieved by the first-order theory. The Surface Evolver results are slightly less accurate, and the second-order theory overestimates the Bond number at which the maximum in $d / \lambda_{c}$ occurs, presumably because the theory is unable to handle Bond numbers less than about 3. Note that, because $\mathrm{Bo}=\left(R / \lambda_{c}\right)^{2}$, for a given value of $\lambda_{c}$ the curves (and symbols) presented in figure $9 \mathrm{~b}$ express a universal relation between $d$ and $R$.

\section{CONCLUSION}

We have measured the shape of an air bubble on the surface of a soap bath, for a range of bubble sizes, corresponding to a range of Bond numbers. Large bubbles, which form for large Bond numbers, are almost perfect hemispheres protruding from the liquid surface, whereas small bubbles, which form for small Bond numbers, are mostly immersed in the

liquid, with only a small lenticular film emerging. We have shown that the Young-Laplace equation can be integrated analytically, in the limit of large Bond numbers, to provide a semi-analytical description of bubble shapes (where a few integrals have to be evaluated numerically at the highest orders) that is in good agreement with both experimental results 
and Surface Evolver calculations, even for not particularly large Bond numbers (Bo $\gtrsim 4)$. In particular we are able to predict the dependence of the meniscus height on the bubble contact angle, a quantity that describes how much the bubble protrudes from the fluid upon which it rests, as its size is changed. Remarkably, this is a non-monotonic function, as already found for $2 \mathrm{~d}$ bubbles by Howell [28]. Our theory is also able to describe how the bubble bottom changes from shallow and flat to deep and curved as the Bond number (and thus the bubble size) is decreased. In particular, we have calculated the bubble depth vs Bond number, which is predicted to reach a maximum for a certain bubble radius. Both the maximum bubble depth and the corresponding bubble radius are a function of the capillary length $\lambda_{c}$ only. Finally, we have shown that, in order to achieve agreement with experiment, it is essential to consider the fully three-dimensional character of the bubble. We have not included the disjoining pressure - the direct interaction between the two film surfaces in our semi-analytical calculations; this is mimicked in the Surface Evolver calculation by using a small (but finite) angle between the inner and outer meniscus surfaces. Since the agreement with experiment and theory remains excellent, we conjecture that in this system its effect is small.

\section{Acknowledgements}

The work of SA was partly supported by the French RENATECH network. SJC thanks K. Brakke for his development and maintenance of the Surface Evolver code and acknowledges funding from the FP7 IAPP project HYDROFRAC (Grant No. PIAP-GA-2009-251475). PICT acknowledges financial support from the Fundação para a Ciência e Tecnologia (Portugal) through contracts nos. EXCL/FIS-NAN/0083/2012 and UID/FIS/00618/2013.

[1] Stevenson, P. Foam Engineering: Fundamentals and Applications; John Wiley \& Sons, 2012.

[2] Cantat, I.; Cohen-Addad, S.; Elias, F.; Graner, F.; Höhler, R.; Pitois, O.; Rouyer, F.; SaintJalmes, A. Foams: Structure and Dynamics; Oxford University Press, 2013.

[3] Sylvester, A.; Döring, T.; Schmidt, A. Liquids, Smoke, and Soap Bubbles: Reflections on Materials for Ephemeral User Interfaces. In Proceedings of the Fourth International Conference 
on Tangible, Embedded, and Embodied Interaction; TEI 10; ACM: New York, NY, USA, 2010; pp 269-270.

[4] Ochiai, Y.; Oyama, A.; Hoshi, T.; Rekimoto, J. Theory and Application of the Colloidal Display: Programmable Bubble Screen for Computer Entertainment. In Advances in Computer Entertainment; Reidsma, D., Katayose, H., Nijholt, A., Eds.; Lecture Notes in Computer Science; Springer International Publishing, 2013; pp 198-214.

[5] Arscott, S. Electrowetting of Soap Bubbles. Appl. Phys. Lett. 2013, 103 (1), 014103.

[6] Amjadi, A.; Feiz, M. S.; Namin, R. M. Liquid Soap Film Generates Electricity: A Suspended Liquid Film Rotating in an External Electric Field as an Electric Generator. Microfluid. Nanofluidics 2014, 18 (1), 141-147.

[7] Feiz, M. S.; Namin, R. M.; Amjadi, A. Theory of the Liquid Film Motor. Phys. Rev. E 2015, $92(3), 033002$.

[8] Yu, G.; Cao, A.; Lieber, C. M. Large-Area Blown Bubble Films of Aligned Nanowires and Carbon Nanotubes. Nat. Nanotechnol. 2007, 2 (6), 372-377.

[9] Georgiou, T.; Britnell, L.; Blake, P.; Gorbachev, R. V.; Gholinia, A.; Geim, A. K.; Casiraghi, C.; Novoselov, K. S. Graphene Bubbles with Controllable Curvature. Appl. Phys. Lett. 2011, $99(9), 093103$.

[10] Zhang, R. Graphene Sponges Prepared from Clusters of Soap Bubbles with Hierarchical Pores. Meet. Abstr. 2014, MA2014-01 (33), 1251.

[11] Boys, C. V. Soap Bubbles: Their Colors and Forces Which Mold Them; Courier Corporation, 1958.

[12] Isenberg, C. The Science of Soap Films and Soap Bubbles; Courier Corporation, 1978.

[13] Hutchings, M.; Morgan, F.; Ritoré, M.; Ros, A. Proof of the Double Bubble Conjecture. Ann. Math. 2002, 155 (2), 459-489.

[14] Goldstein, R. E.; Moffatt, H. K.; Pesci, A. I.; Ricca, R. L. Soap-Film Möbius Strip Changes Topology with a Twist Singularity. Proc. Natl. Acad. Sci. 2010, 107 (51), 21979-21984.

[15] Bonhomme, O.; Liot, O.; Biance, A.-L.; Bocquet, L. Soft Nanofluidic Transport in a Soap Film. Phys. Rev. Lett. 2013, 110 (5), 054502.

[16] Moulton, D. E.; Pelesko, J. A. Theory and Experiment for Soap-Film Bridge in an Electric Field. J. Colloid Interface Sci. 2008, 322 (1), 252-262.

[17] Rodrigues, J. F.; Saramago, B.; Fortes, M. A. Apparent Contact Angle and Triple-Line Tension 
of a Soap Bubble on a Substrate. J. Colloid Interface Sci. 2001, 239 (2), 577-580.

[18] Teixeira, P. I. C.; Fortes, M. A. Line Tension and Excess Energy of a Wall Plateau Border. Phys. Rev. E 2007, 75 (1), 011404.

[19] Teixeira, M. A. C.; Teixeira, P. I. C. Contact Angle of a Hemispherical Bubble: An Analytical Approach. J. Colloid Interface Sci. 2009, 338 (1), 193-200.

[20] Arscott, S. Wetting of Soap Bubbles on Hydrophilic, Hydrophobic, and Superhydrophobic Surfaces. Appl. Phys. Lett. 2013, 102 (25), 254103.

[21] Bird, J. C.; de Ruiter, R.; Courbin, L.; Stone, H. A. Daughter Bubble Cascades Produced by Folding of Ruptured Thin Films. Nature 2010, 465 (7299), 759-762.

[22] Kim, D.; Yi, S. J.; Kim, H. D.; Kim, K. C. Visualization Study on the Transient Liquid Film Behavior and Inner Gas Flow after Rupture of a Soap Bubble. J. Vis. 2014, 17 (4), 337-344.

[23] Bico, J. Cracks in Bursting Soap Films. J. Fluid Mech. 2015, 7r8, 1-4.

[24] Scarano, F.; Ghaemi, S.; Caridi, G. C. A.; Bosbach, J.; Dierksheide, U.; Sciacchitano, A. On the Use of Helium-Filled Soap Bubbles for Large-Scale Tomographic PIV in Wind Tunnel Experiments. Exp. Fluids 2015, 56 (2), 1-12.

[25] Kornek, U.; Müller, F.; Harth, K.; Hahn, A.; Ganesan, S.; Tobiska, L.; Stannarius, R. Oscillations of Soap Bubbles. New J. Phys. 2010, 12 (7), 073031.

[26] Afanasyev, Y. D.; Andrews, G. T.; Deacon, C. G. Measuring Soap Bubble Thickness with Color Matching. Am. J. Phys. 2011, 79 (10), 1079-1082.

[27] Nicolson, M. M. The interaction between floating particles. Math. Proc. Camb. Philos. Soc. 1949, $45(02), 288-295$.

[28] Howell, P. D. The draining of a two-dimensional bubble. J. Engng. Maths. 1999, 35, 251-272.

[29] Harkins, W. D.; Brown, F. E. The determination of surface tension (free surface energy), and the weight of falling drops: the surface tension of water and benzene by the capillary weight method. J. Am. Chem. Soc. 1919, 41 (4), 499-524.

[30] Lee, B.-B.; Ravindra, P.; Chan, E.-S. A critical review: surface and interfacial tension measurement by the drop weight method. Chem. Eng. Commun. 2008, 195 (3), 889-924.

[31] Brakke, K. The Surface Evolver. Exp. Math. 1992, 1 (2), 141-165 (see also http://facstaff.susqu.edu/brakke/evolver/evolver.html). 
(a)

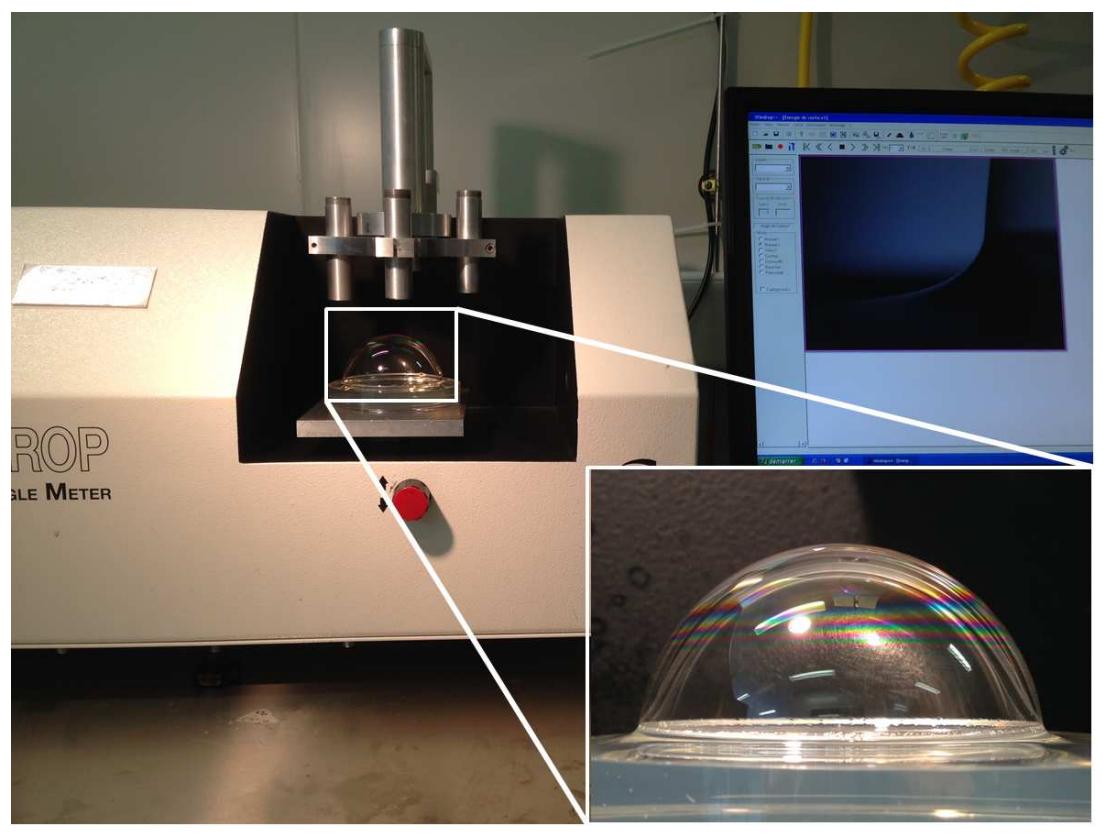

(b)

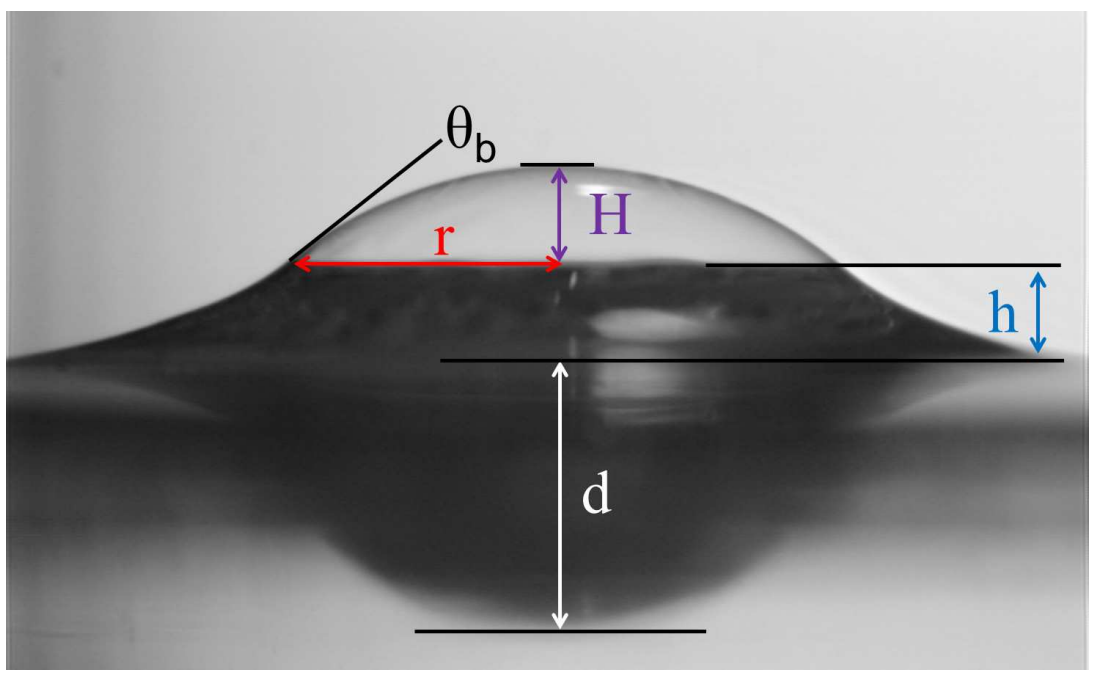

FIG. 1: (a) Experimental set-up for measuring the bubble parameters. (b) Experimentallymeasured bubble parameters: contact angle of the bubble $\left(\theta_{b}\right)$, radius of the top of the meniscus or base of the bubble $(r)$ (related to the bubble film curvature radius $R$ through $r=R \sin \theta_{b}$ ), bubble height measured from the top of the meniscus $(H)$, meniscus height $(h)$ and bubble depth $(d)$. See the text for details. 


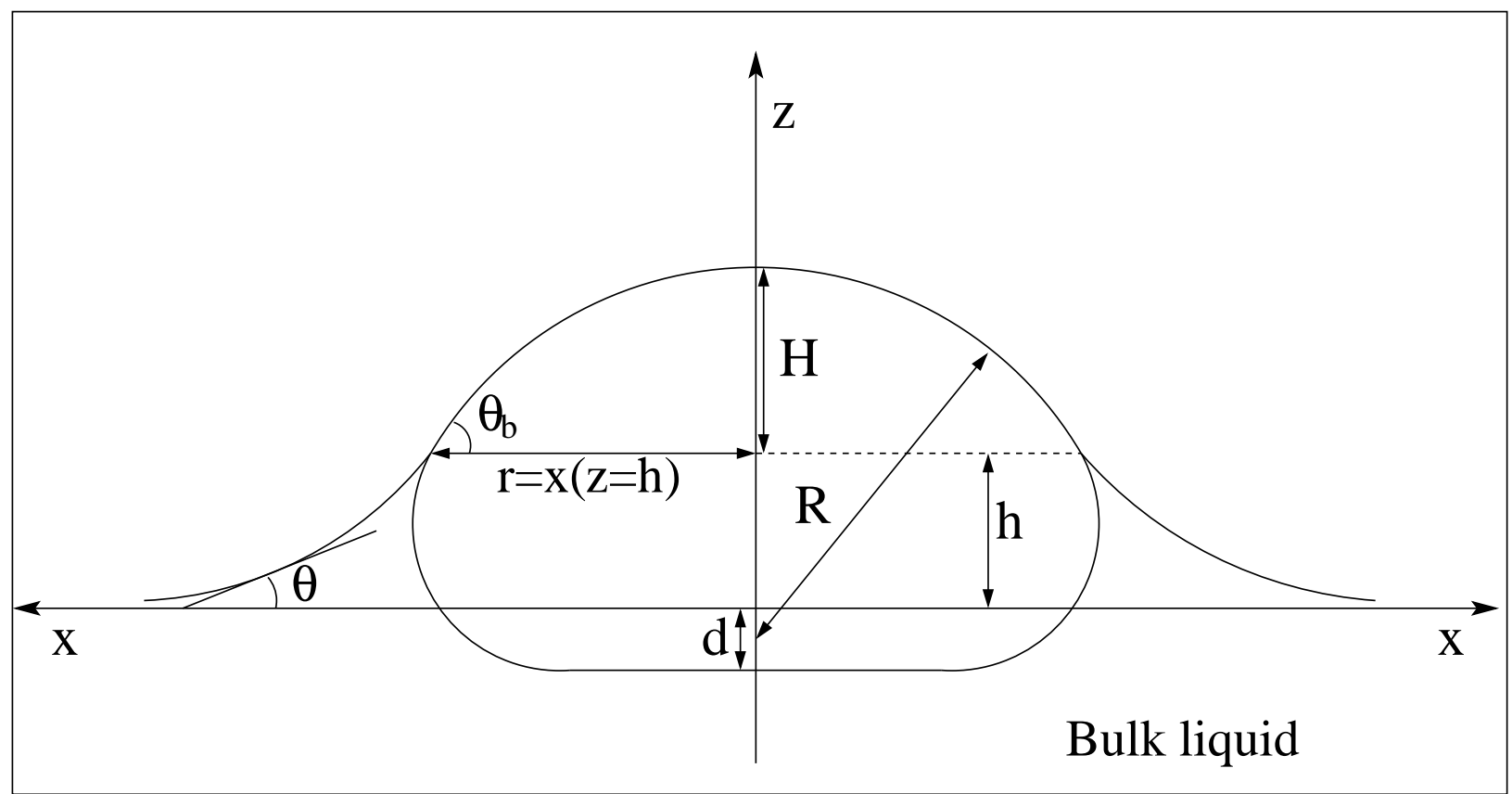

FIG. 2: Sketch of a bubble and the associated meniscus on a free liquid surface. $R$ is the bubble film curvature radius, $d$ is the bubble depth, $H$ is the bubble height measured from the top of the meniscus, $h$ is the meniscus height, $\theta$ is the film/meniscus inclination, and $\theta_{b}$ is the contact angle of the bubble (film inclination at the top of the meniscus). $z$ is the height and $x$ is the radial distance from the film to the axis of symmetry of the bubble (the $z$-axis). The $z=0$ plane lies on the free liquid surface infinitely far from the bubble. Note that $x$ is a radial coordinate, and that on the meniscus surfaces, i.e., for $z<h, 0<\theta<\theta_{b}$ outside the bubble and $\theta_{b}<\theta<\pi$ inside the bubble. 


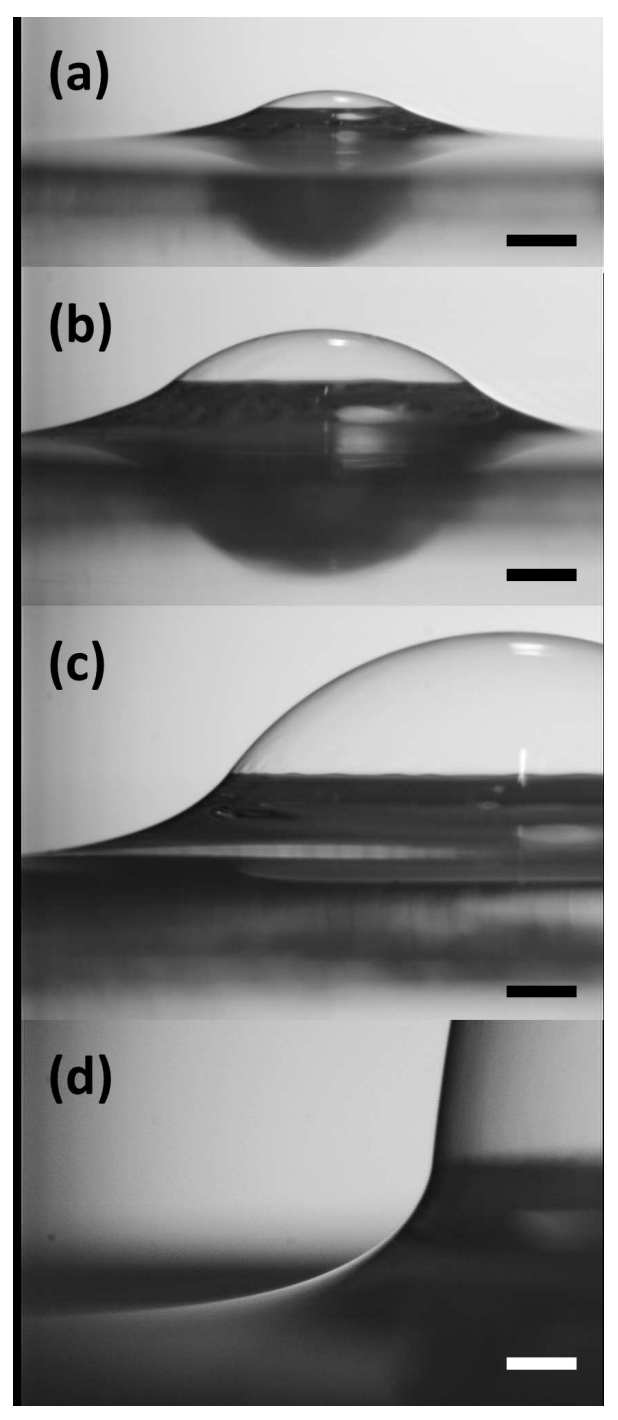

FIG. 3: Photographs of sessile bubbles resting on a bath of soap solution. The radii at the top of the meniscus and contact angles are approximately (a) $r=1 \mathrm{~mm}, \theta_{b}=25.7^{\circ}$; (b) $r=2.1 \mathrm{~mm}$, $\theta_{b}=38.5^{\circ} ;$ (c) $r=4.2 \mathrm{~mm}, \theta_{b}=52.5^{\circ}$; and (d) $r=32.5 \mathrm{~mm}, \theta_{b}=83.9^{\circ}$. The scale bar corresponds to $1 \mathrm{~mm}$. The surface tension of the soap solution is approximately $28.2 \mathrm{~mJ} \mathrm{~m}{ }^{-2}$. 


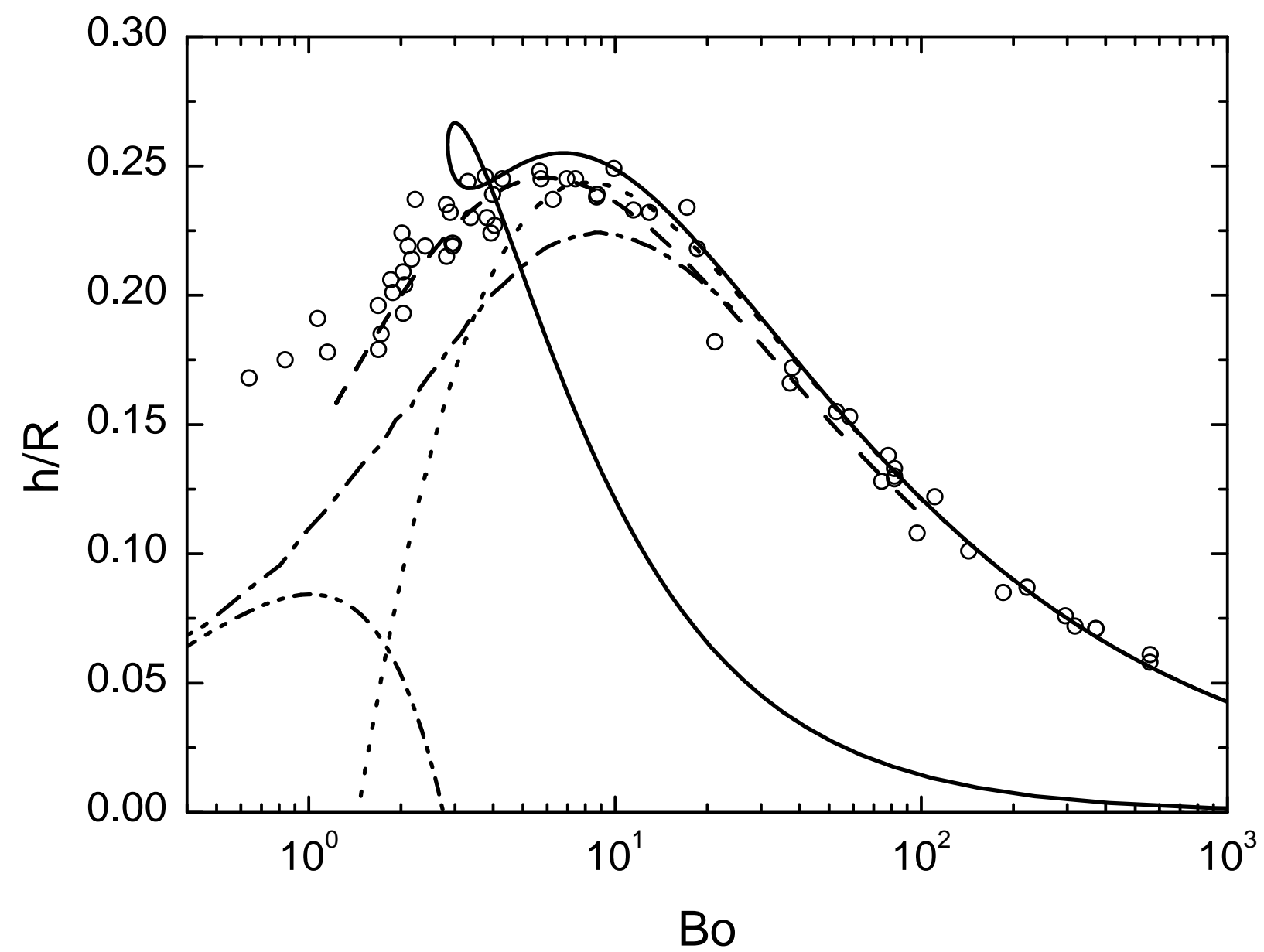

FIG. 4: Dimensionless meniscus height $h / R$ vs Bond number Bo. Symbols: experimental results; solid line: second-order analytical theory; dotted line: first-order analytical theory; dashed line: Surface Evolver results; dash-dotted line: Howell's 2d solution; dash-double-dotted line: Howell's $3 \mathrm{~d}$ solution for small Bo [28]. Because of the wide range of Bond numbers in the measurements, the horizontal axis is logarithmic. 


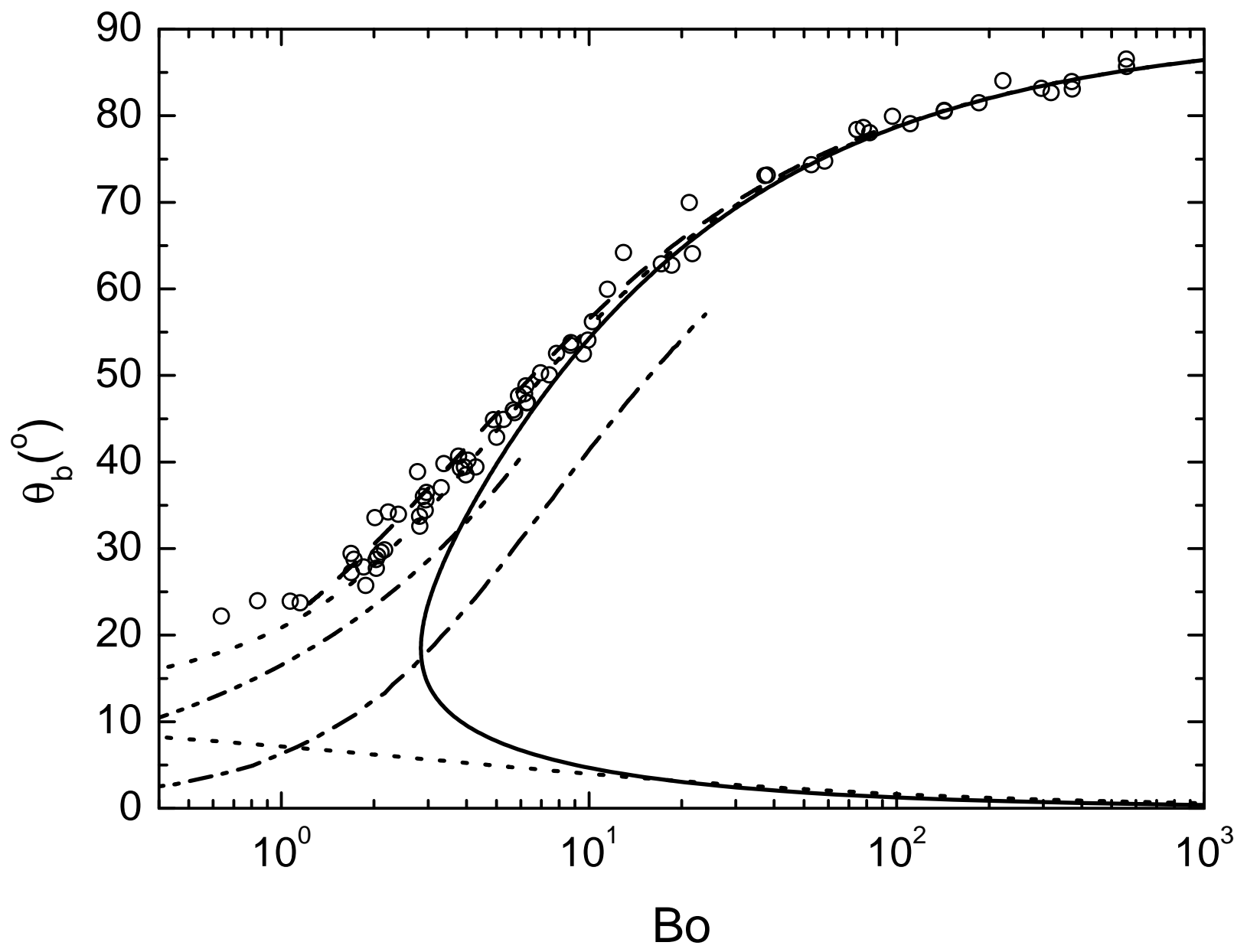

FIG. 5: Apparent contact angle $\theta_{b}$ vs Bond number Bo. Symbols as in figure 4. 
(a)

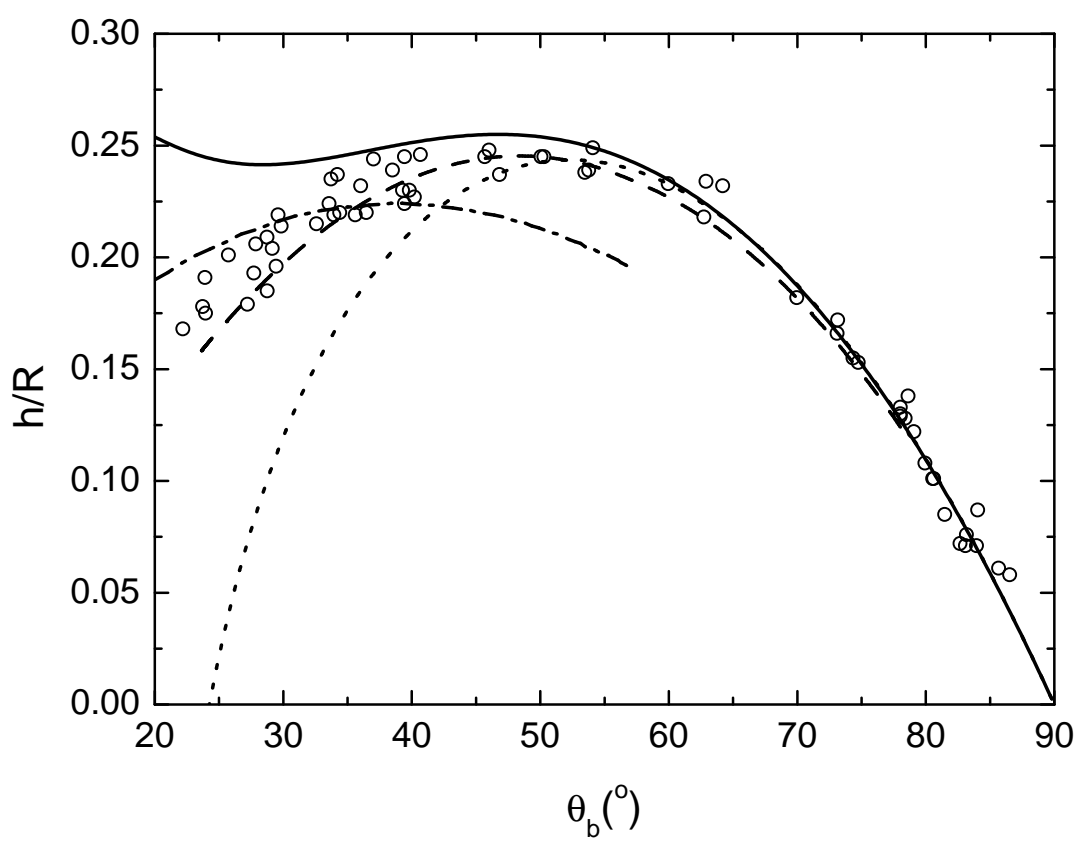

(b)

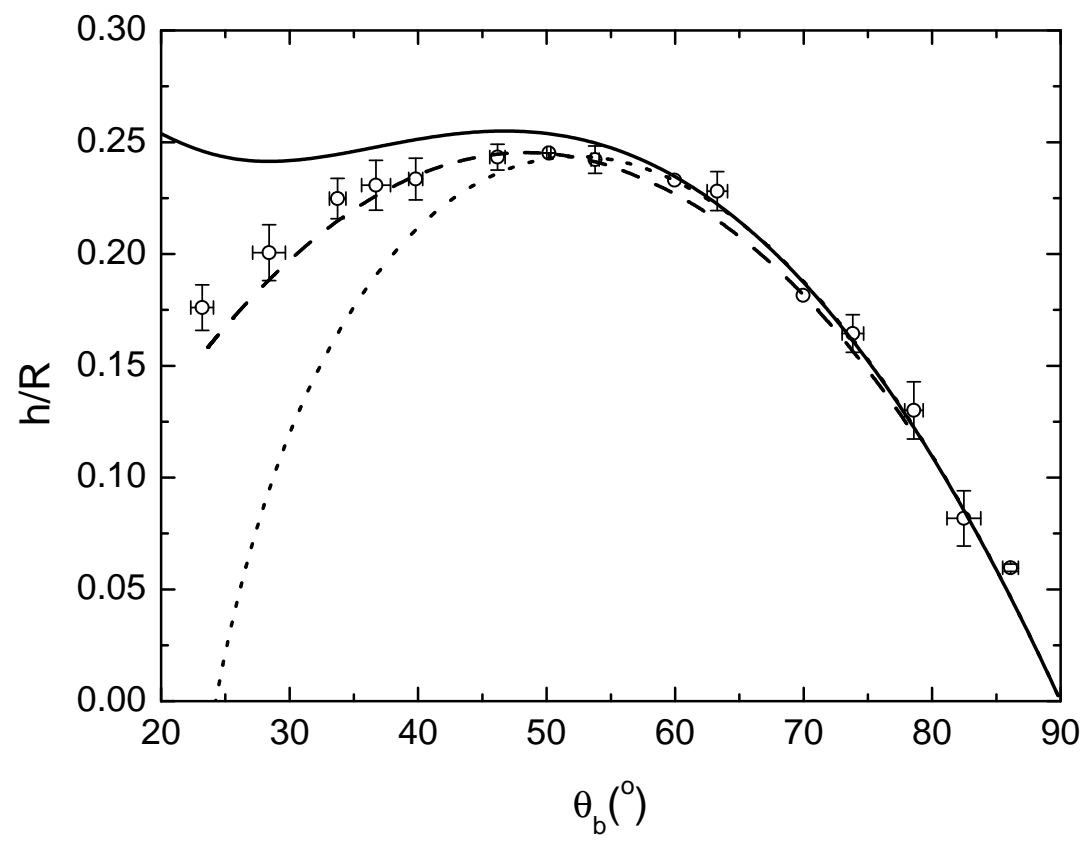

FIG. 6: (a) Dimensionless meniscus height $h / R$ vs bubble contact angle $\theta_{b}$. Symbols: experimental results; solid line: second-order analytical theory; dotted line: first-order analytical theory; dashed line: Surface Evolver results; dash-dotted line: Howell's 2d solution [28]. (b) Binned data in (a) with error bars (see section II for details), compared with our first- and second-order approximations, and Surface Evolver results. 

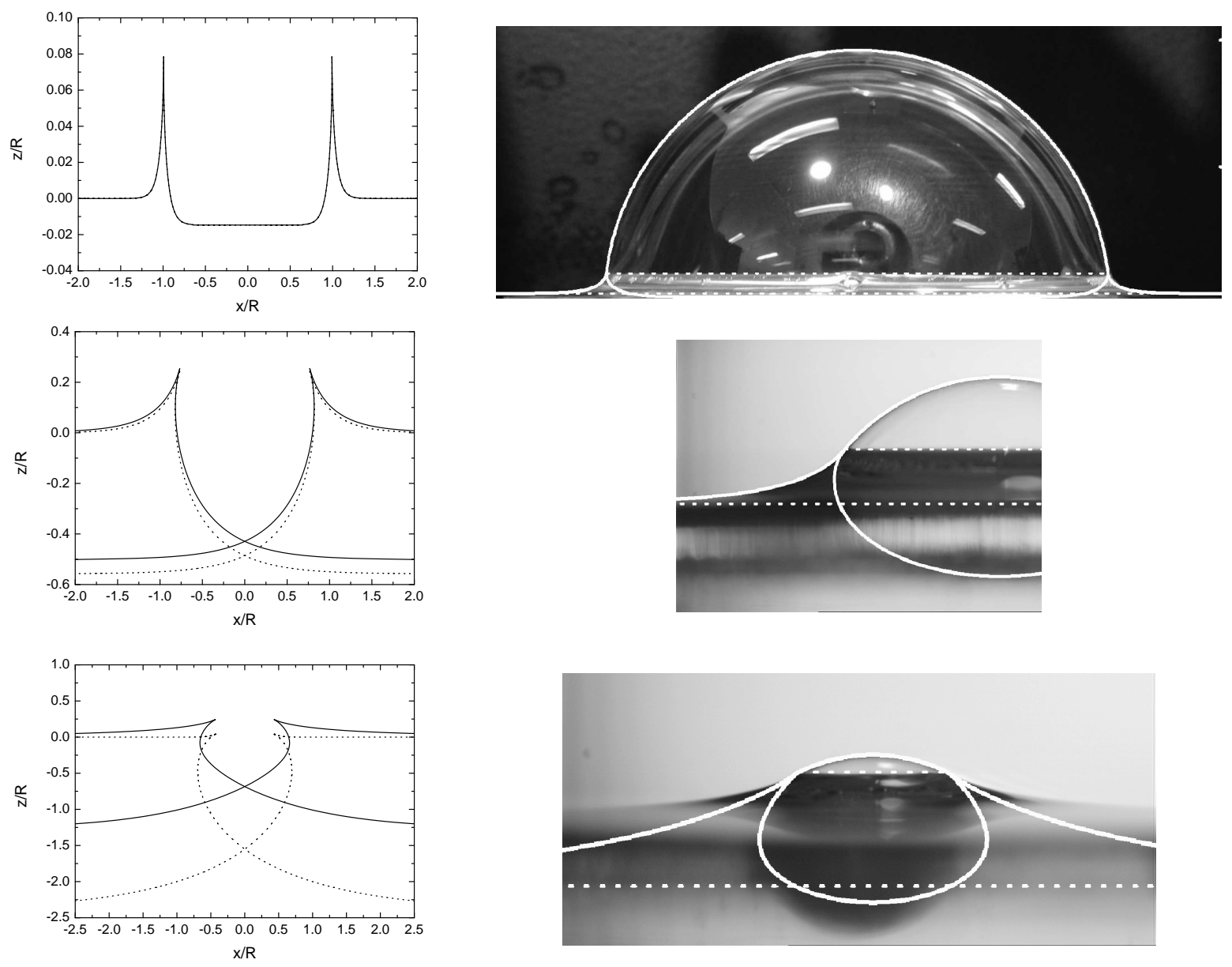

FIG. 7: Full bubble profiles for a large bubble (large Bo, $\theta_{b}=83.16^{\circ}$, top row), an intermediate bubble (moderate Bo, $\theta_{b}=50.06^{\circ}$, middle row) and a small bubble (small Bo, $\theta_{b}=25.74^{\circ}$, bottom row). The left column shows the first-order (dotted lines) and second-order (solid lines) inner and outer solutions for the menisci on both sides of the bubble, where $x$ and $z$ have both been re-scaled by $R$. Here $x$ is the standard abscissa ranging from $-\infty$ to $+\infty$. The right column shows the sum of the second-order inner and outer solutions for the two menisci plus the bubble film, overlaid on photographs of actual bubbles with the same $\theta_{b}$. 


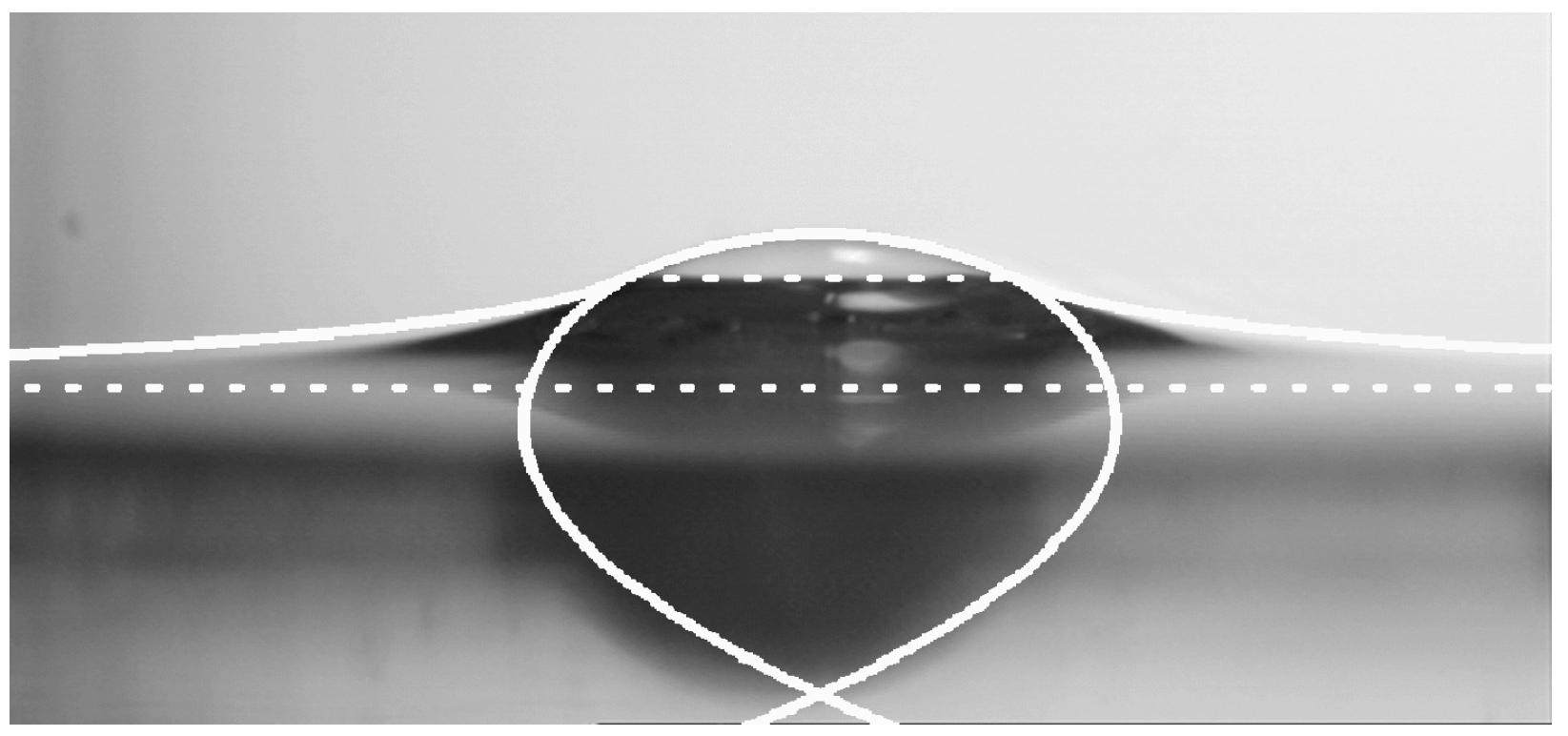

FIG. 8: Same as in figure 7, lower right, but now the solutions for the menisci on either side of the bubble have not been added together. Overall agreement is better, although at the expense of a sharp - and unrealistic - vertex at the bottom of the bubble. 
(a)

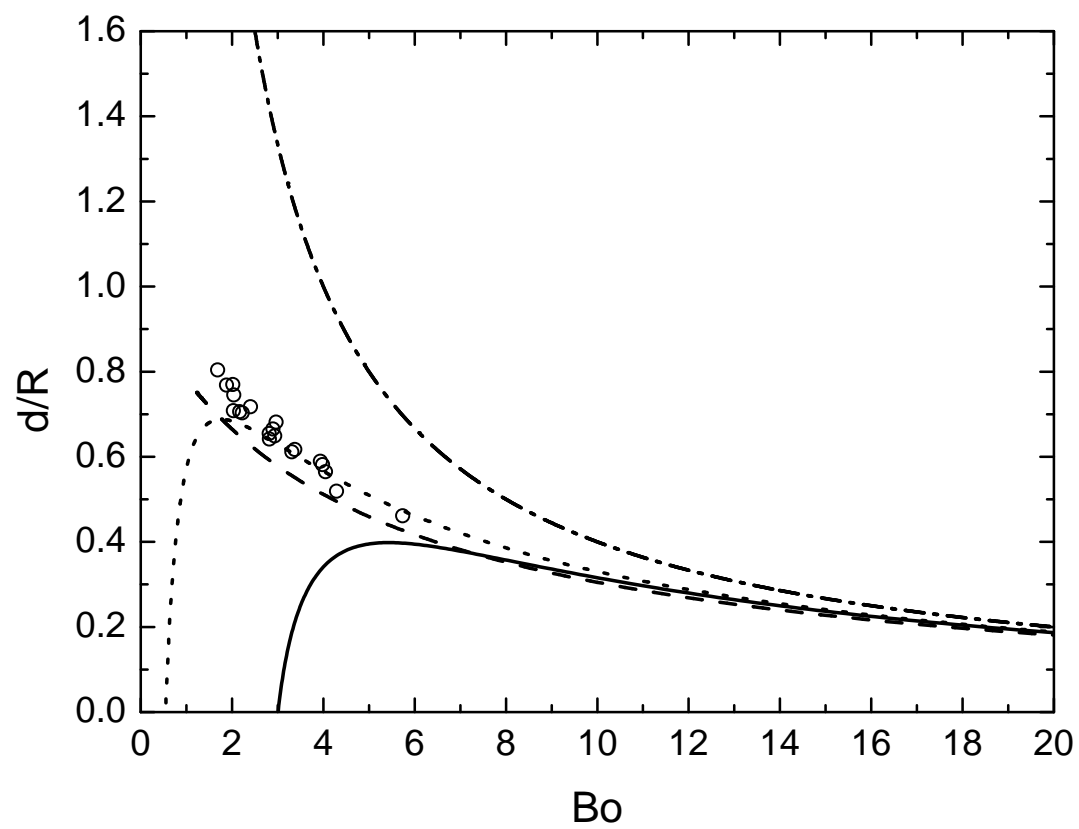

(b)

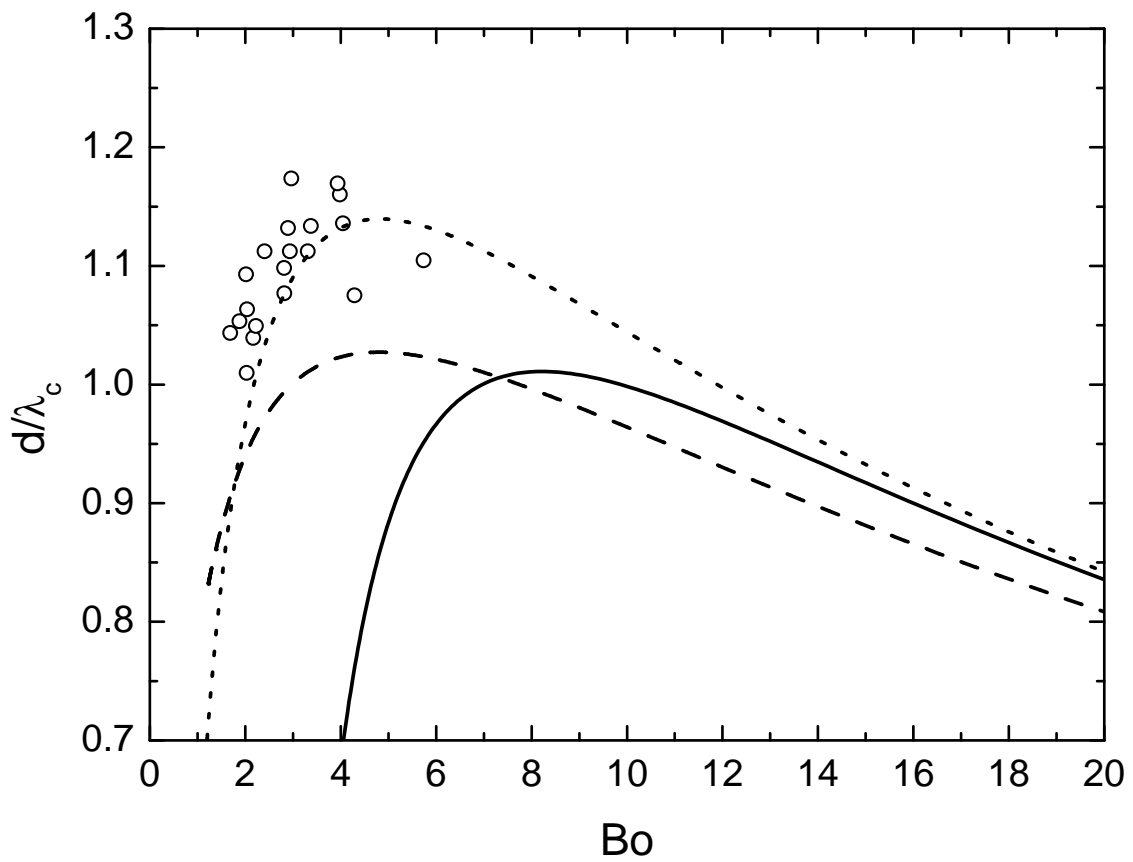

FIG. 9: (a) Dimensionless bubble depth $d / R$ vs Bond number. Symbols: experimental data; solid line: second-order analytical theory; dotted line: first-order analytical theory (both obtained from the profile constructed by adding the solutions for the two menisci); dashed line: Surface Evolver results; dash-dotted line: analytical theory using equation (42). See the text for details of calculation. (b) Scaled bubble depth $d / \lambda_{c}$ vs Bond number. Symbols as in (a). Note that the Bond number dependence can be converted to a bubble radius dependence using equation (10). 
Figure for Table of Contents

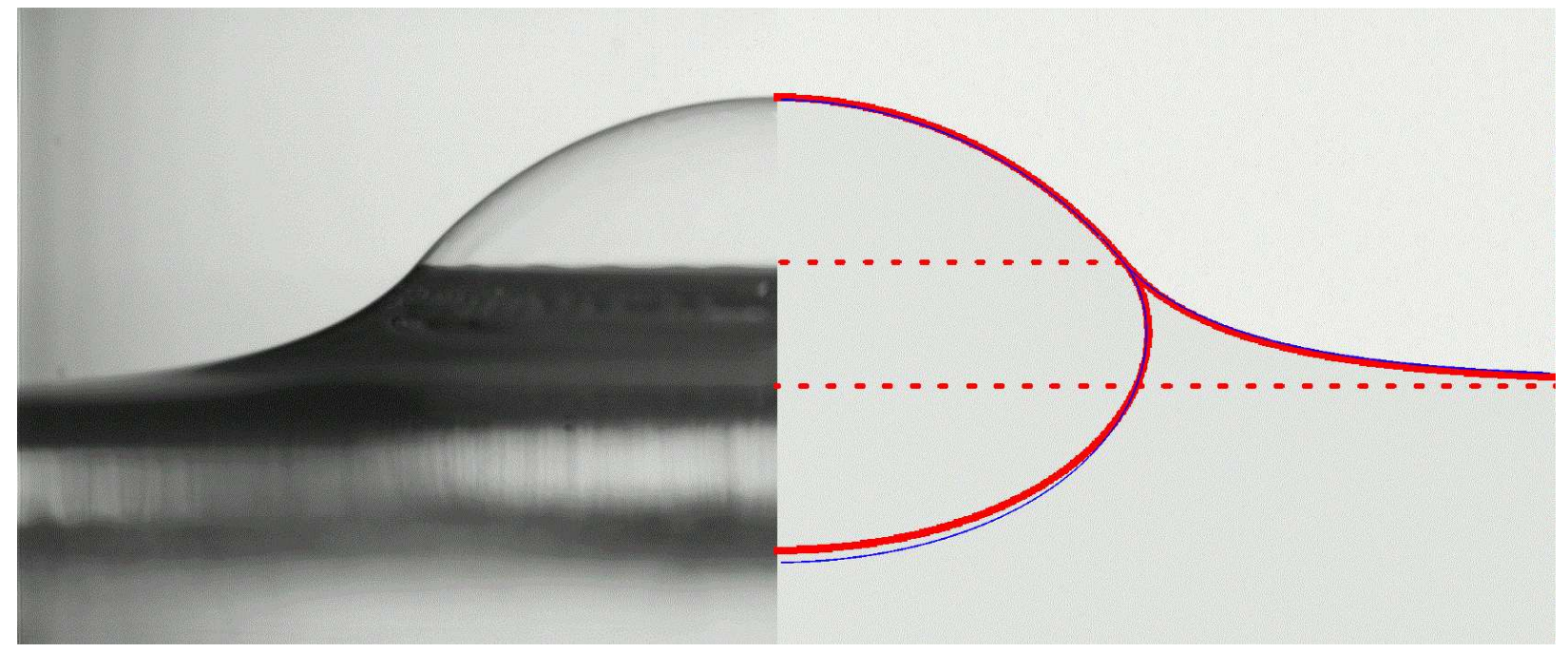

